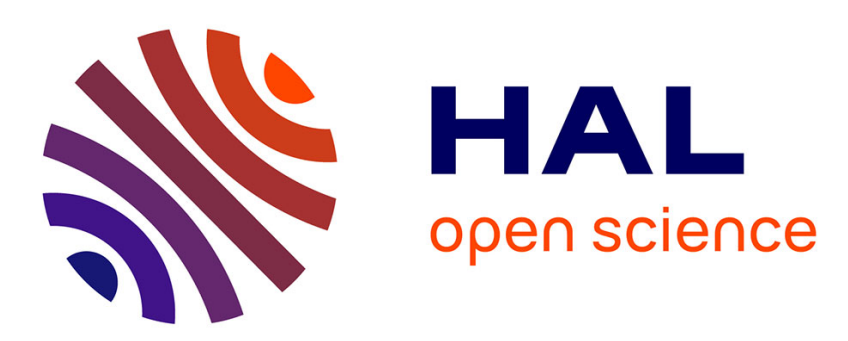

\title{
Weighted coloring on planar, bipartite and split graphs: complexity and improved approximation
}

\author{
Dominique de Werra, Marc Demange, Bruno Escoffier, Jérôme Monnot, \\ Vangelis Th. Paschos
}

\section{- To cite this version:}

Dominique de Werra, Marc Demange, Bruno Escoffier, Jérôme Monnot, Vangelis Th. Paschos. Weighted coloring on planar, bipartite and split graphs: complexity and improved approximation. xxx, 2004, France. pp.896-907. hal-00004074

\section{HAL Id: hal-00004074 https://hal.science/hal-00004074}

Submitted on 26 Jan 2005

HAL is a multi-disciplinary open access archive for the deposit and dissemination of scientific research documents, whether they are published or not. The documents may come from teaching and research institutions in France or abroad, or from public or private research centers.
L'archive ouverte pluridisciplinaire HAL, est destinée au dépôt et à la diffusion de documents scientifiques de niveau recherche, publiés ou non, émanant des établissements d'enseignement et de recherche français ou étrangers, des laboratoires publics ou privés. 


\title{
Weighted coloring on planar, bipartite and split graphs: complexity and approximation
}
D. de Werra *
M. Demange ${ }^{\dagger}$
B. Escoffier ${ }^{\ddagger}$
J. Monnot ${ }^{\ddagger}$
V. Th. Paschos ${ }^{\ddagger}$

\begin{abstract}
We study complexity and approximation of MIN WEIGHTED NODE COLORING in planar, bipartite and split graphs. We show that this problem is NP-complete in planar graphs, even if they are triangle-free and their maximum degree is bounded above by 4 . Then, we prove that Min Weighted NOde COLORING is NP-complete in $P_{8}$-free bipartite graphs, but polynomial for $P_{5}$-free bipartite graphs. We next focus ourselves on approximability in general bipartite graphs and improve earlier approximation results by giving approximation ratios matching inapproximability bounds. We next deal with MIN WEIGHTED EDGE COLORING in bipartite graphs. We show that this problem remains strongly NP-complete, even in the case where the input-graph is both cubic and planar. Furthermore, we provide an inapproximability bound of $7 / 6-\varepsilon$, for any $\varepsilon>0$ and we give an approximation algorithm with the same ratio. Finally, we show that MIN WEIGHTED NODE COLORING in split graphs can be solved by a polynomial time approximation scheme.

Keywords: Graph coloring; ; weighted node coloring; weighted edge coloring; approximability; NP-completeness; planar graphs; bipartite graphs; split graphs.
\end{abstract}

\section{Introduction}

We give in this paper some complexity results as well as some improved approximation results for Min Weighted NODE COLORING, originally studied in Guan and Zhu [7] and more recently in [4]. A $k$-coloring of $G=(V, E)$ is a partition $\mathcal{S}=\left(S_{1}, \ldots, S_{k}\right)$ of the node set $V$ of $G$ into stable sets $S_{i}$. In this case, the objective is to determine a node coloring minimizing $k$. A natural generalization of this problem is obtained by assigning a strictly positive integer weight $w(v)$ for any node $v \in V$, and defining the weight of stable set $S$ of $G$ as $w(S)=\max \{w(v): v \in S\}$. Then, the objective is to determine $\mathcal{S}=\left(S_{1}, \ldots, S_{k}\right)$ a node coloring of $G$ minimizing the quantity $\sum_{i=1}^{k} w\left(S_{i}\right)$. This problem is easily shown NP-hard; it suffices to consider $w(v)=1, \forall v \in V$ and MiN WeIGHTED NODE COLORING becomes the classical node coloring problem. Other versions of weighted colorings have been studied in Hassin and Monnot [8].

\footnotetext{
${ }^{*}$ Ecole Polytechnique Fédérale de Lausanne, Switzerland, dewerra@ima.epfl.ch

${ }^{\dagger}$ ESSEC, Dept. SID, France, demange@essec.fr

${ }^{\ddagger}$ LAMSADE, Université Paris-Dauphine, France, \{escoffier, monnot, paschos\}@lamsade.dauphine.fr
} 
Consider an instance $I$ of an NP-hard optimization problem $\Pi$ and a polynomial time algorithm A computing feasible solutions for $\Pi$. Denote by $m_{\mathrm{A}}(I, S)$ the value of a $\Pi$-solution $S$ computed by A on $I$ and by $\operatorname{opt}(I)$, the value of an optimal $\Pi$-solution for $I$. The quality of $\mathrm{A}$ is expressed by the ratio (called approximation ratio in what follows) $\rho_{\mathrm{A}}(I)=m_{\mathrm{A}}(I, S) / \operatorname{opt}(I)$, and the quantity $\rho_{\mathrm{A}}=\inf \left\{r: \rho_{\mathrm{A}}(I)<r, I\right.$ instance of $\left.\Pi\right\}$. A very favourable situation for polynomial approximation occurs when an algorithm achieves ratios bounded above by $1+\varepsilon$, for any $\varepsilon>0$. We call such algorithms polynomial time approximation schemes. The complexity of such schemes may be polynomial or exponential in $1 / \varepsilon$ (they are always polynomial in the sizes of the instances). A polynomial time approximation scheme with complexity polynomial also in $1 / \varepsilon$ is called fully polynomial time approximation scheme.

This paper extends results on MIN WEIGHTED NODE COLORING, the study of which has started in Demange et al. [4]. We first deal with planar graphs and we show that, for this family, the problem studied is NP-complete, even if we restrict to triangle-free planar graphs with node-degree not exceeding 4 .

We then deal with particular families of bipartite graphs. The NP-completeness of Min WEIGHTED NODE COLORING has been established in [4] for general bipartite graphs. We show here that this remains true even if we restrict to planar bipartite graphs or to $P_{21}$-free bipartite graphs (for definitions graph-theoretical notions used in this paper, the interested reader is referred to Berge [1]). It is interesting to observe that these results are obtained as corollaries of a kind of generic reduction from the precoloring extension problem shown to be NP-complete in Bodlaender et al. [2], Hujter and Tuza [10,11], Kratochvil [13]. Then, we slightly improve the last result to $P_{8}$-free bipartite graphs and show that the problem becomes polynomial in $P_{5}$-free bipartite graphs. Observe that in [4], we have proved that MIN WEIGHTED NODE COLORING is polynomial for $P_{4}$-free graphs and NP-complete for $P_{5}$-free graphs.

Then, we focus ourselves on approximability of MIN WEIGHTED NODE COLORING in (general) bipartite graphs. As proved in [4], this problem is approximable in such graphs within approximation ratio $4 / 3$; in the same paper a lower bound of $8 / 7-\varepsilon$, for any $\varepsilon>0$, was also provided. Here we improve the approximation ratio of [4] by matching the 8/7-lower bound of [4] with a same upper bound; in other words, we show here that MIN WEIGHTED NODE COLORING in bipartite graphs is approximable within approximation ratio bounded above by $8 / 7$.

We next deal with MiN WEIGHTED EDGE COLORING in bipartite graphs. In this problem we consider an edge-weighted graph $G$ and try to determine a partition of the edges of $G$ into matchings in such a way that the sum of the weights of these matchings is minimum (analogously to the nodemodel, the weight of a matching is the maximum of the weights of its edges). In [4], it is shown that Min WEIGHTED EDGE COLORING is NP-complete for cubic bipartite graphs. Here, we slightly strengthen this result showing that this problem remains strongly NP-complete, even in cubic and planar bipartite graphs. Furthermore, we strengthen the inapproximability bound provided in [4], by reducing it from $8 / 7-\varepsilon$ to $7 / 6-\varepsilon$, for any $\varepsilon>0$. Also, we match it with an upper bound of the same value, improving so the $5 / 3$-approximation ratio provided in [4].

Finally, we deal with approximation of MIN WEIGHTED NODE COLORING in split graphs. As 
proved in [4], MIN Weighted NODE COLORING is strongly NP-complete in such graphs, even if the nodes of the input graph receive only one of two distinct weights. It followed that this problem cannot be solved by fully polynomial time approximation schemes, but no approximation study was addressed there. In this paper we show that MIN WEIGHTED NODE COLORING in split graphs can be solved by a polynomial time approximation scheme.

In the remainder of the paper we shall assume for any weighted node or edge coloring $\mathcal{S}=$ $\left(S_{1}, \ldots, S_{\ell}\right)$ considered, we will have $w\left(S_{1}\right) \geqslant \ldots \geqslant w\left(S_{\ell}\right)$.

\section{$2 \quad$ Weighted node coloring in triangle-free planar graphs}

The node coloring problem in planar graphs has been shown NP-complete by Garey and Johnson [5], even if the maximum degree does not exceed 4. On the other hand, this problem becomes easy in triangle-free planar graphs, (see Grotzsch [6]). Here, we show that the weighted node coloring problem is NP-complete in triangle-free planar graphs with maximum degree 4 by using a reduction from 3-SAT PLANAR, proved to be NP-complete in Lichtenstein [14]. This problem is defined as follows: Given a collection $\mathcal{C}=\left(C_{1}, \ldots, C_{m}\right)$ of clauses over the set $X=\left\{x_{1}, \ldots, x_{n}\right\}$ of Boolean variables such that each clause $C_{j}$ has at most three literals (and at least two), is there a truth assignment $f$ satisfying $\mathcal{C}$ ? Moreover, the bipartite graph $B P=(L, R ; E)$ is planar where $|L|=n$, $|R|=m$ and $\left[x_{i}, c_{j}\right] \in E$ iff the variable $x_{i}\left(\right.$ or $\overline{x_{i}}$ ) appears in the clause $C_{j}$.

Theorem 2.1 Min Weighted NOdE COLORING is $\mathbf{N P}$-complete in triangle-free planar graphs with a maximum degree 4.

Proof : Let $B P=(L, R ; E)$ be the bipartite graph representing an instance $(X, \mathcal{C})$ of 3-SAT PLANAR where $L=\left\{x_{1}, \ldots, x_{n}\right\}, R=\left\{c_{1}, \ldots, c_{m}\right\}$. We construct an instance $I=(G, w)$ of Min WEIGHTED NODE COLORING by using two gadgets: The gadgets clause $F\left(C_{j}\right)$ are given in Figure 1 for clause $C_{j}$ of size 3 and in Figure 2 for clause $C_{j}$ of size 2. The nodes $c_{j}^{k}$ are those that will be linked to the rest of the graph.

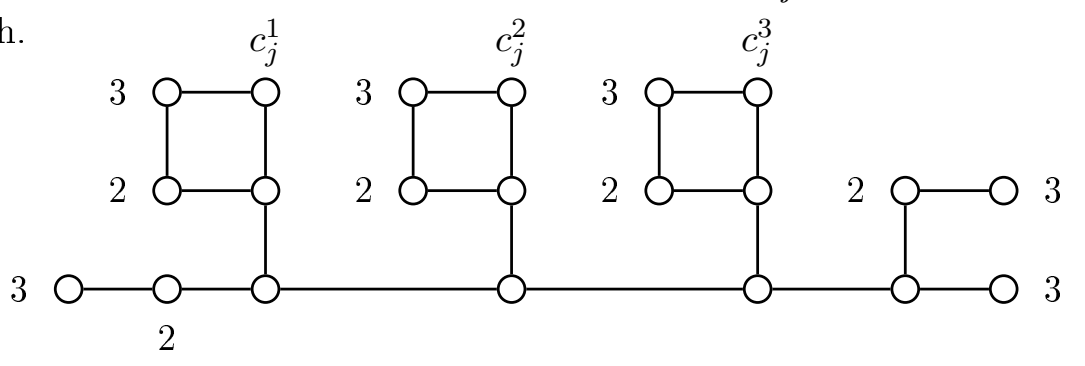

Figure 1: Graph $F\left(C_{j}\right)$ representing a clause $C_{j}$ of size 3 .

The gadgets variable $H\left(x_{i}\right)$ is given in Figure 3 for variable $x_{i}$. Assume that $x_{i}$ appears $p_{1}$ times positively and $p_{2}$ times negatively in $(X, \mathcal{C})$, then in $H\left(x_{i}\right)$ there are $2 p=2\left(p_{1}+p_{2}\right)$ special nodes $x_{i}^{k}, \overline{x_{i}^{k}}, k=1, \ldots, p$. These nodes form a path which meets nodes $x_{i}^{k}, \overline{x_{i}^{k}}$ alternately. 


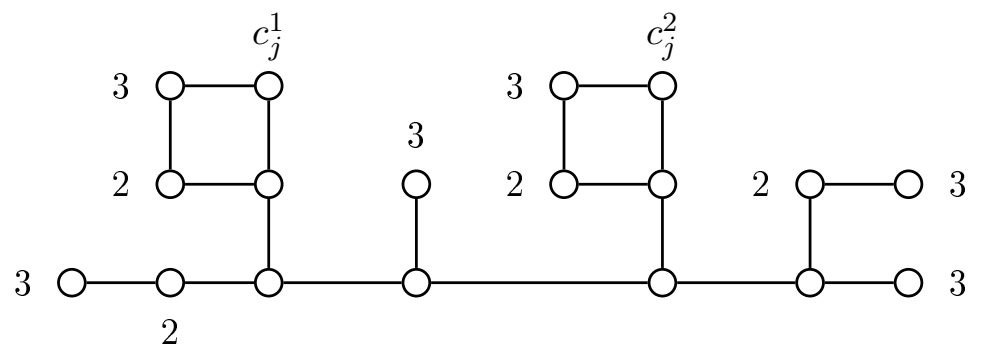

Figure 2: Graph $F\left(C_{j}\right)$ representing a clause $C_{j}$ of size 2 .

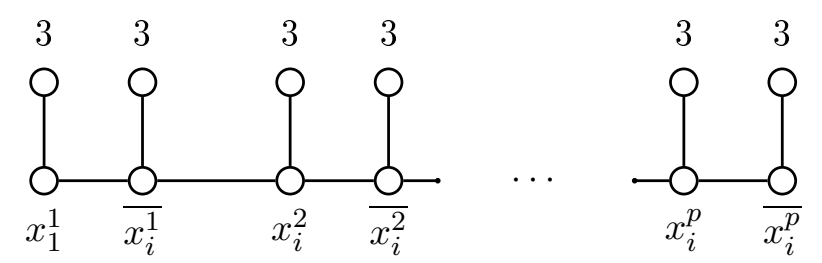

Figure 3: Graph $H\left(x_{i}\right)$ representing variable $x_{i}$

The weight of nodes which are not given in Figures 1,2 and 3 are 1. These gadgets are linked together by the following process. If variable $x_{i}$ appears positively (resp. negatively) in clause $c_{j}$, we link one of the variables $\overline{x_{i}^{k}}$ (resp. $x_{i}^{k}$ ), with a different $k$ for each $C_{j}$, to one of the three nodes $c_{j}^{l}$ of gadget $F\left(C_{j}\right)$. This can be done in a way which preserves the planarity of the graph. Observe that $G$ is triangle-free and planar with maximum degree 4 . Moreover, we assume that $G$ is not bipartite (otherwise, we add a disjoint cycle $\Gamma$ with $|\Gamma|=7$ and $\forall v \in V(\Gamma), w(v)=1$ ).

It is then not difficult to check that $(X, \mathcal{C})$ is satisfiable iff $\operatorname{opt}(I) \leqslant 6$.

\section{$3 \quad$ Weighted node coloring in bipartite graphs}

\subsection{Complexity results}

The NP-completeness of MIN WEIGHTED NODE COLORING in bipartite graphs has been proved in [4]. Here, we show that some more restrictive versions are also NP-complete, namely bipartite planar graphs and $P_{8}$-free bipartite graphs, i.e. bipartite graphs which do not contain induced paths of length 8 or more. We use a generic reduction from the precoloring extension node coloring problem (in short PrExt node COLORING). This latter problem studied in $[2,10,13,11]$, can be described as follows. Given a positive integer $k$, a graph $G=(V, E)$ and $k$ pairwise disjoint subsets $V_{1}, \ldots, V_{k}$ of $V$, we want to decide if there exists a node coloring $\mathcal{S}=\left(S_{1}, \ldots, S_{k}\right)$ of $G$ such that $V_{i} \subseteq S_{i}$, for all $i \leqslant k$. Moreover, we restrict to some class of graphs $\mathcal{G}$ : we assume that $\mathcal{G}$ is closed when we add a pending edge with a new node (i.e., if $G=(V, E) \in \mathcal{G}$ and $x \in V, y \notin V$, then $G+[x, y] \in \mathcal{G}$ ).

Theorem 3.1 Let $\mathcal{G}$ be a class of graphs which is closed when we add a pending edge with a new node. If PREXT NODE COLORING is $\mathbf{N P}$-complete for graphs in $\mathcal{G}$, then MiN WEIGHTED NOdE 


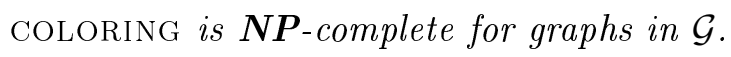

Proof : Let $\mathcal{G}$ be such a class of graphs. We shall reduce PREXT NOdE COLORING in $\mathcal{G}$ graphs to weighted node coloring in $\mathcal{G}$ graphs. Let $G=(V, E) \in \mathcal{G}$ and $k$ pairwise disjoint subsets $V_{1}, \ldots, V_{k}$ of $V$. We build instance $I=\left(G^{\prime}, w\right)$ of weighted node coloring using several gadgets $T_{i}$, for $i=$ $1, \ldots, k$. The construction of $T_{i}$ is given by induction as follows: $T_{1}$ is simply a root $v_{1}$ with weight $w\left(v_{1}\right)=2^{k-1}$. Given $T_{1}, \ldots, T_{i-1}, T_{i}$ is a tree with a root $v_{i}$ of weight $w\left(v_{i}\right)=2^{k-i}$ that we link to tree $T_{p}$ via edge $\left[v_{i}, v_{p}\right]$ for each $p=1, \ldots, i-1$.
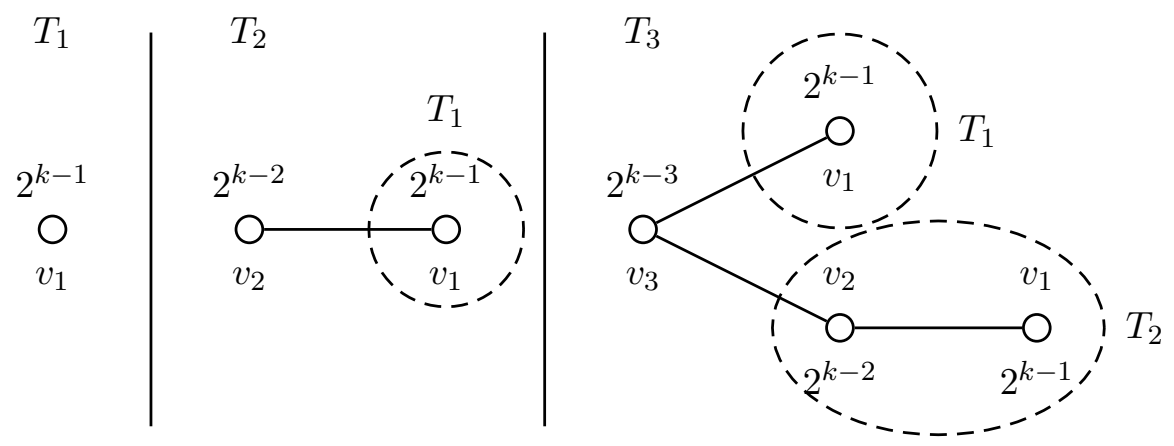

Figure 4: Gadgets for $T_{1}, T_{2}$ and $T_{3}$.

Figure 4 illustrates the gadgets $T_{1}, T_{2}, T_{3}$. Now, $I=\left(G^{\prime}, w\right)$ where $G^{\prime}=\left(V^{\prime}, E^{\prime}\right)$ is constructed in the following way: $G^{\prime}$ contains $G$. For all $i=1, \ldots, k$, we replace each node $v \in V_{i}$ by a copy of the gadget $T_{i}$ where we identify $v$ with root $v_{i}$. For all $v \in V \backslash\left(\cup_{i=1}^{k} V_{i}\right)$ we set $w(v)=1$. Note that, by hypothesis, $G^{\prime} \in \mathcal{G}$.

One can verify that the precoloring of $G$ (given by $V_{1}, \ldots, V_{k}$ ) can be extended to a proper node coloring of $G$ using at most $k$ colors iff $\operatorname{opt}(I) \leqslant 2^{k}-1$.

Using the results of Kratochvil [13] on the NP-completeness of PREXT NODE COLORING in bipartite planar graphs for $k=3$ and $P_{13}$-free bipartite graphs for $k=5$, we deduce:

Corollary 3.2 In bipartite planar graphs, MIN WEIGHTED NODE COLORING is strongly NP-complete and it is not $\frac{8}{7}-\varepsilon$-approximable unless $\boldsymbol{P}=\boldsymbol{N P}$.

Corollary 3.3 In $P_{21}$-free bipartite graphs, MIN WEIGHTED NODE COLORING is strongly $\mathbf{N P}$-complete and it is not $\frac{32}{31}-\varepsilon$-approximable unless $\boldsymbol{P}=\boldsymbol{N P}$.

In Hujter and Tuza [11], it is shown that PrExt NODE COLORING is NP-complete in $P_{6}$-free bipartite chordal graphs for unbounded $k$. Unfortunately, we cannot use this result in Theorem 3.1 since the resulting graph has an induced path with arbitrarily large length. However, we can adapt their reduction.

Theorem 3.4 min Weighted NOde COloring is $\mathbf{N P}$-complete in $P_{8}$-free bipartite graphs.

Proof : We shall reduce 3-SAT-3, proved to be NP-complete in Papadimitriou [16] to our problem. Given a collection $\mathcal{C}=\left(C_{1}, \ldots, C_{m}\right)$ of clauses over the set $X=\left\{x_{1}, \ldots, x_{n}\right\}$ of Boolean variables such 
that each clause $C_{j}$ has at most three literals and each variable has at most 3 occurrences (2 positive and one negative), we construct an instance $I=(B P, w)$ in the following way: we start from $B P_{1}=$ $\left(L_{1}, R_{1} ; E_{1}\right)$, a complete bipartite graph $K_{n, m}$ where $L_{1}=\left\{x_{1}, \ldots, x_{n}\right\}$ and $R_{1}=\left\{c_{1}, \ldots, c_{m}\right\}$. Moreover, each node of $B P_{1}$ has weight 1 . There is also another bipartite graph $B P_{2}$ isomorphic to $K_{2 n, 2 n}$ where a perfect matching has been deleted. More formally, $B P_{2}=\left(L_{2}, R_{2} ; E_{2}\right)$ where $L_{2}=\left\{l_{1}, \ldots, l_{2 n}\right\}, R_{2}=\left\{r_{1}, \ldots, r_{2 n}\right\}$ and $\left[l_{i}, r_{j}\right] \in E_{2}$ iff $i \neq j$. Finally, $w\left(l_{i}\right)=w\left(r_{i}\right)=2^{2 n-i}$ for $i=1, \ldots, 2 n$. Indeed, sets $\left\{l_{2 i-1}, r_{2 i-1}\right\}$ and $\left\{l_{2 i}, r_{2 i}\right\}$ will correspond to literal $x_{i}$ and $\overline{x_{i}}$ respectively. Between $B P_{1}$ and $B P_{2}$, there is a set $E_{3}$ of edges. $\left[x_{i}, r_{j}\right] \notin E_{3}$ iff $j=2 i-1$ or $j=2 i$ and $\left[l_{i}, c_{j}\right] \notin E_{3}$ iff $i=2 k-1$ and $x_{k}$ is in $C_{j}$ or $i=2 k$ and $\overline{x_{k}}$ is in $C_{j}$. Note that $B P$ is a $P_{8}$-free bipartite graph. One can verify that $(X, \mathcal{C})$ is satisfiable iff $\operatorname{opt}(I) \leqslant 2^{2 n}-1$.

We end this section by stating that MIN WEIGHTED NODE COLORING is polynomial for $P_{5}$-free bipartite graphs, i.e., without induced chain on 5 nodes. There are several characterizations of $P_{5}$-free bipartite graphs, see for example, Hammer et al. [9], Chung et al. [3] and Hujter and Tuza [10]. In particular, $B P$ is a $P_{5}$-free bipartite graph iff $B P$ is bipartite and each connected component of $B P$ is $2 K_{2}$-free, i.e., its complement is $C_{4}$-free. In this case, we can show that any optimal weighted node coloring $\mathcal{S}^{*}=\left(S_{1}^{*}, \ldots, S_{\ell}^{*}\right)$ uses at most 3 colors (so, $\ell \leqslant 3$ ) and when $\ell=3$, then for any connected component $B P_{i}=\left(L_{i}, R_{i} ; E_{i}\right)$ of $P_{5}$-free bipartite graph we have $S_{1}^{*, i} \cap L_{i} \neq \emptyset$ and $S_{1}^{*, i} \cap R_{i} \neq \emptyset$, $S_{2}^{*, i} \subset R_{i}$ (resp., $S_{2}^{*, i} \subset L_{i}$ ) and $S_{3}^{*, i} \subset L_{i}$ (resp., $S_{3}^{*, i} \subset R_{i}$ ) where $\left(S_{1}^{*, i}, S_{2}^{*, i}, S_{3}^{*, i}\right)$ is the restriction of $\mathcal{S}^{*}$ to the subgraph $B P_{i}$. Thus, applying an exhaustive search on $k_{1}=w\left(S_{2}^{*}\right)$ and a dichotomy search $k_{2}=w\left(S_{3}^{*}\right)$ we can find an optimal solution within $O(n|w| \log |w|)$ time where $|w|=|\{w(v): v \in V\}|$. Hence, we can state:

Theorem 3.5 MIN WEIGHTED NODE COLORING is polynomial in $P_{5}$-free bipartite graphs and can be solved within time $O(n|w| \log |w|)$.

\subsection{Approximation}

In Demange et al. [4], a $\frac{4}{3}$-approximation is given for Min WEIGHTED NODE COLORING and it is proved that a $\left(\frac{8}{7}-\varepsilon\right)$-approximation is not possible, for any $\varepsilon>0$, unless $\mathbf{P}=\mathbf{N P}$, even if we consider arbitrarily large values of opt $(I)$. Using Corollary 3.2, we deduce that this lower bound also holds if we consider bipartite planar graphs. Here, we give a $\frac{8}{7}$-approximation in bipartite graphs.

\section{BIPARTITECOLOR}

1 Sort the nodes in non-increasing weight order (i.e., $w\left(v_{1}\right) \geqslant \ldots \geqslant w\left(v_{n}\right)$ );

2 For $i=1$ to $n$ do

2.1 Set $V_{i}=\left\{v_{1}, \ldots, v_{i}\right\}$

2.2 Compute $\mathcal{S}_{i}^{*}=\left(S_{1}^{i}, S_{2}^{i}\right)$ ( $S_{2}^{i}$ may be empty) an optimal weighted node 2-coloring in the subgraph $B P\left[V_{i}\right]$ induced by $V_{i}$;

2.3 Define node coloring $\mathcal{S}^{i}=\left(S_{1}^{i}, S_{2}^{i}, L \backslash V_{i}, R \backslash V_{i}\right)\left(L \backslash V_{i}\right.$ or/and $R \backslash V_{i}$ may be empty); 
3 Output $\mathcal{S}=\operatorname{argmin}\left\{\operatorname{val}\left(\mathcal{S}^{i}\right): i=1, \ldots, n\right\}$

The step 2.2 consists of computing the (unique) 2-coloration $\left(S_{1, j}^{*}, S_{2, j}^{*}\right)\left(\right.$ with $\left.w\left(S_{1, j}^{*}\right) \geqslant w\left(S_{2, j}^{*}\right)\right)$ of each connected component $B P_{j}, j=1 \ldots p$ of $B P\left[V_{i}\right]$ (with $S_{2, j}^{*}=\emptyset$ if $B P_{j}$ is an isolated node). Then it merges the most expensive sets, i.e. it computes $S_{1}^{i}=\cup_{j=1}^{p} S_{i, j}^{*}$ for $i=1,2$. It is easy to observe that $\mathcal{S}_{i}^{*}=\left(S_{1}^{i}, S_{2}^{i}\right)$ is the best weighted node coloring of $B P\left[V_{i}\right]$ among the colorings using at most 2 colors; such a coloring can be found in $O(m)$ time where $m=|E|$.

Theorem 3.6 BIPARTITECOLOR polynomially solves in time $O(n m)$ MIN WEIGHTED NODE COLORING in bipartite-graphs and it is a $\frac{8}{7}$-approximation.

Proof : Let $I=(B P, w)$ be a weighted bipartite-graph where $B P=(L, R ; E)$ and $\mathcal{S}^{*}=\left(S_{1}^{*}, \ldots, S_{l}^{*}\right)$ be an optimal node coloring of $I$ with $w\left(S_{1}^{*}\right) \geq \ldots \geq w\left(S_{l}^{*}\right)$. If $l<3$, then BIPARTITECOLOR finds an optimal weighted node coloring which is $\mathcal{S}^{n}$. Now, assume $l \geqslant 3$ and let $i_{j}=\min \left\{k: v_{k} \in S_{j}^{*}\right\}$. We have $i_{1}=1$ and $\operatorname{opt}(I) \geqslant w\left(v_{i_{1}}\right)+w\left(v_{i_{2}}\right)+w\left(v_{i_{3}}\right)$.

Let us examine several steps of this algorithm. When $i=i_{2}-1$, the algorithm produces a node 3-coloring $\mathcal{S}^{i_{2}-1}=\left(S_{i_{2}-1}^{1}, L \backslash S_{i_{2}-1}^{1}, R \backslash S_{i_{2}-1}^{1}\right)$. Indeed, by construction $V_{i_{2}-1} \subseteq S_{1}^{*}$ is an independent set, and then, $\mathcal{S}_{i_{2}-1}^{*}$ is defined by $S_{1}^{i_{2}-1}=V_{i_{2}-1}, S_{2}^{i_{2}-1}=\emptyset$ and then $\operatorname{val}\left(\mathcal{S}^{i_{2}-1}\right) \leqslant$ $w\left(v_{i_{1}}\right)+2 w\left(v_{i_{2}}\right)$. When $i=i_{3}-1$, the algorithm produces on $B P\left[V_{i_{3}-1}\right]$ a node 2-coloring $\mathcal{S}_{i_{3}-1}^{*}$ with a cost $\operatorname{val}\left(\mathcal{S}_{i_{3}-1}^{*}\right) \leqslant w\left(v_{i_{1}}\right)+w\left(v_{i_{2}}\right)$ since the coloring $\left(S_{1}^{*} \cap V_{i_{3}-1}, S_{2}^{*} \cap V_{i_{3}-1}\right)$ is a feasible node 2-coloring of $B P\left[V_{i_{3}-1}\right]$ with cost $w\left(v_{i_{1}}\right)+w\left(v_{i_{2}}\right)$. Thus, $\operatorname{val}\left(\mathcal{S}^{i_{3}-1}\right) \leqslant w\left(v_{i_{1}}\right)+w\left(v_{i_{2}}\right)+2 w\left(v_{i_{3}}\right)$. Finally, when $i=n$, the node 2-coloring $\mathcal{S}^{n}$ satisfies $\operatorname{val}\left(\mathcal{S}^{n}\right) \leqslant 2 w\left(v_{i_{1}}\right)$

The convex combination of these 3 values with coefficients $\frac{1}{7} \times \operatorname{val}\left(\mathcal{S}^{n}\right), \frac{4}{7} \times \operatorname{val}\left(\mathcal{S}^{i_{3}-1}\right)$ and $\frac{2}{7} \times \operatorname{val}\left(\mathcal{S}^{i_{2}-1}\right)$ gives the expected result.

\section{$4 \quad$ Weighted edge coloring in bipartite graphs}

The weighted edge coloring problem on a graph $G$ can be viewed as the weighted node coloring problem on $L(G)$ where $L(G)$ is the line graph of $G$. Here, for simplicity, we refer to the edge model.

\subsection{Complexity results}

Demange et al. [4] have proved that MIN WEIGHTED EDGE COLORING in bipartite cubic graphs is strongly NP-complete and a lower bound of $\frac{8}{7}$ is given for the approximation. Here, we slightly improve these complexity results.

Theorem 4.1 In bipartite cubic planar graphs, MIN WEIGHTED EDGE COLORING is strongly NPcomplete and it is not $\frac{7}{6}-\varepsilon$-approximable unless $\boldsymbol{P}=\mathbf{N P}$.

Proof : We shall reduce PREXT EDGE COLORING in bipartite cubic planar graphs to our problem. Given a bipartite cubic planar graph $B P$ and 3 pairwise disjoint matchings $E_{i}$, the question of PREXT EDGE COLORING is to determine if it is possible to extend the edge precoloring $E_{1}, E_{2}, E_{3}$ 
to a proper 3-edge coloring of $G$. Very recently, this problem has been shown NP-complete in Marx [15].

Let $B P=(V, E)$ and $E_{1}, E_{2}, E_{3}$ be an instance of PREXT EDGE COLORING; we construct an instance $I=\left(B P^{\prime}, w\right)$ of weighted edge coloring as follows. Each edge in $E_{1}$ receives weight 3 . Each edge $[x, y] \in E_{2}$ is replaced by a gadget $F_{2}$ described in Figure 4.1, where we identify $x$ and $y$ to $v_{0}$ and $v_{9}$ respectively. Each edge in $E_{3}$ is replaced by a gadget $F_{3}$ which is the same as gadget $F_{2}$ except that we have exchanged weights 1 and 2 . The other edges of $G$ receive weight 1 . Remark that $B P^{\prime}$ is still a bipartite cubic planar graph.

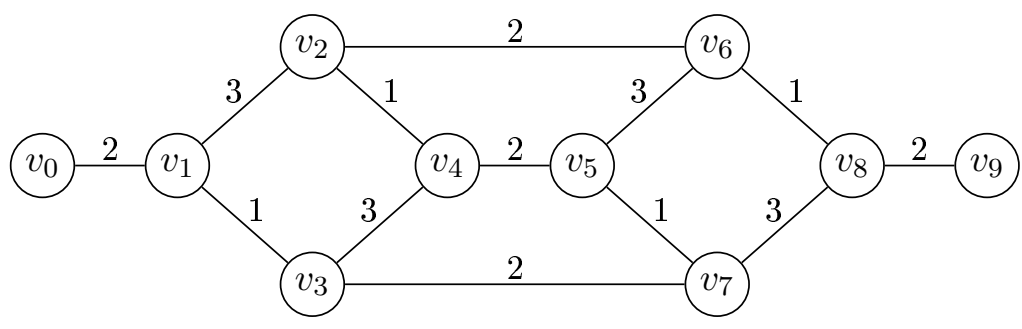

Figure 5: Gadget $F_{2}$ for $e \in E_{2}$.

We can verify that the answer of PREXT EDGE COLORING instance is yes if and only if there exists an edge coloring $\mathcal{S}$ of $I$ with cost $\operatorname{val}(\mathcal{S}) \leqslant 6$.

\subsection{Approximation}

In Demange et al. [4], a $\frac{5}{3}$-approximation is given for MIN WEIGHTED EDGE COLORING in bipartite graphs with maximum degree 3 . Here, we give a $\frac{7}{6}$-approximation. We need some notations: If $B P=(V, E)$ is a bipartite graph with node set $V=\left\{v_{1}, \ldots, v_{n}\right\}$, we always assume that its edges $E=\left\{e_{1}, \ldots, e_{m}\right\}$ are sorted in non-increasing weight order (i.e., $\left.w\left(e_{1}\right) \geqslant \ldots \geqslant w\left(e_{m}\right)\right)$. If $V^{\prime}$ is a subset of nodes and $E^{\prime}$ a subset of edges, $B P\left[V^{\prime}\right]$ and $B P\left[E^{\prime}\right]$ denote the subgraph of $B P$ induced by $V^{\prime}$ and the partial graph of $B P$ induced by $E^{\prime}$ respectively. For any $i \leqslant m$, we set $E_{i}=\left\{e_{1}, \ldots, e_{i}\right\}$ and $\overline{E_{i}}=E \backslash E_{i}$. Finally, $V_{i}$ denotes the set of nodes of $B P$ incident to an edge in $E_{i}$ (so, it is the subset of non-isolated nodes of $\left.B P\left[E_{i}\right]\right)$.

\section{BIPARTITEEDGECOLOR}

1 For $i=m$ downto 1 do

1.1 Apply algorithm SOL1 on $B P\left[E_{i}\right]$;

1.2 If $\operatorname{SOL} 1\left(B P\left[E_{i}\right]\right) \neq \emptyset$, complete in a greedy way all the colorings produced by SOL1 on the edges of $\overline{E_{i}}$. Let $\mathcal{S}_{1, i}$ be a best one among these edge colorings of $B P$;

1.3 For $j=i$ downto 1 do

1.3.1 Apply algorithm SOL2 on $B P\left[E_{j}\right]$; 
1.3.2 If SOL2 $\left(B P\left[E_{j}\right]\right) \neq \emptyset$, complete in a greedy way all the colorings produced by SOL2 on the edges of $\overline{E_{j}}$. Let $\mathcal{S}_{2, j, i}$ be a best one among these edge colorings of $B P$;

1.3.3 Apply algorithm SOL3 on $B P\left[E_{j}\right]$;

1.3.4 If $\operatorname{SOL} 3\left(B P\left[E_{j}\right]\right) \neq \emptyset$, complete in a greedy way all the colorings produced by SOL3 on the edges of $\overline{E_{j}}$. Let $\mathcal{S}_{3, j, i}$ be a best one among these edge colorings of $B P$

2 Output $\mathcal{S}=\operatorname{argmin}\left\{\operatorname{val}\left(\mathcal{S}_{1, i}\right), \operatorname{val}\left(\mathcal{S}_{k, j, i}\right): k=2,3, j=1, \ldots, i, i=1, \ldots, m\right\}$

The greedy steps 1.2, 1.2.2 and 1.2.4 give a solution using at most 5 colors. More generally, in [4], we have proved that, in any graph $G$, the greedy coloring and at least one optimal weighted node coloring use at most $\Delta(G)+1$ colors, where $\Delta(G)$ denotes the maximum degree of $G$. In our case, we have $G=L(H)$, the line graph of $H$, and we deduce $\Delta(L(H))+1 \leqslant 2(\Delta(H)-1)+1=2 \Delta(H)-1$. The 3 algorithms SOL1, SOL2 and SOL3 are used on several partial graphs $B P^{\prime}$ of $B P$. In the following, $V^{\prime}, E^{\prime}$ and $m^{\prime}$ denote respectively the node set, the edge set and the number of edge of the current graph $B P^{\prime}$. Moreover, we set $\overline{V_{i}^{\prime}}=V^{\prime} \backslash V_{i}^{\prime}$. If $M=\left(M_{1}, \ldots, M_{l}\right)$ with $w\left(M_{1}\right) \geqslant \ldots \geqslant w\left(M_{\ell}\right)$ is an edge coloring of $B P^{\prime}$, we note $i_{j}=\min \left\{k: e_{k} \in M_{j}\right\}$. We assume, for reason of readability, that some colors $M_{j}$ may be empty (in this case $i_{j}=m^{\prime}+1$ ). The principle of theses algorithms consist in finding a decomposition of $B P^{\prime}$ (a subgraph of $B P$ ) into two subgraphs $B P_{1}^{\prime}$ and $B P_{2}^{\prime}$ having each a maximum degree 2 . When there exists such a decomposition, we can color $B P_{i}^{\prime}$ with at most 2 colors since $B P$ is bipartite.

SOL1

1 For $j=m^{\prime}$ downto 1 do

1.1 If the degree of $B P^{\prime}\left[E_{j}^{\prime}\right]$ is at most 2 then

1.1.1 Consider the graph $B P^{\prime j}$ induced by the nodes of $B P^{\prime}$ incident to at least 2 edges of $\overline{E_{j}^{\prime}}$ and restricted to the edges of $\overline{E_{j}^{\prime}}$.

1.1.2 Determine if there exists a matching $M^{j}$ of $B P^{\prime j}$ such that every node of $\overline{V_{j}^{\prime}}$ is saturated;

1.1.3 If such a matching is found, consider the decomposition $B P_{1, j}^{\prime}$ and $B P_{2, j}^{\prime}$ of $B P^{\prime}$ induced by $E_{j}^{\prime} \cup M^{j}$ and $E^{\prime} \backslash\left(E_{j}^{\prime} \cup M^{j}\right)$ respectively;

1.1.4 Find an optimal 2-edge coloring $\left(M_{1}^{j}, M_{2}^{j}\right)$ of $B P_{1, j}^{\prime}$;

1.1.5 Color greedily the edges of $B P_{2, j}^{\prime}$ with two colors $\left(M_{3}^{j}, M_{4}^{j}\right)$;

1.1.6 Define $\mathcal{S}_{1}^{j}=\left(M_{1}^{j}, M_{2}^{j}, M_{3}^{j}, M_{4}^{j}\right)$ the edge coloring of $B P^{\prime}$;

2 Output $\left\{\mathcal{S}_{1}^{j}: j=1, \ldots, m^{\prime}-1\right\}$ 
Note that the step 1.1.2 is polynomial. Indeed, more generally, given a graph $G$ and $V^{\prime} \subseteq V$, it is polynomial to determine if there exists a matching such that each node of $V^{\prime}$ is matched. To see this, consider $G^{\prime}$ where we add to $G$ all missing edges between nodes of $V \backslash V^{\prime}$. If $|V|$ is odd, then we add a node to the clique $V \backslash V^{\prime}$. It is easy to see that $G^{\prime}$ has a perfect matching if and only if $G$ has a matching such that each node of $V^{\prime}$ is saturated.

Lemma 4.2 If $\mathcal{S}=\left(M_{1}, M_{2}, M_{3}, M_{4}\right)$ is an edge coloring of $B P^{\prime}$, then we have val $\left(\mathcal{S}_{1}^{i_{3}-1}\right) \leqslant$ $w\left(M_{1}\right)+w\left(M_{2}\right)+2 w\left(M_{3}\right)$.

SOL2

1 For $k=m^{\prime}$ downto 1 do

1.1 If $E_{k}^{\prime}$ is a matching :

1.1.1 Determine if there exists a matching $M_{k}$ of $B P^{\prime}\left[\overline{V_{k}^{\prime}}\right]$ such that each node of $B P^{\prime}\left[\overline{V_{k}^{\prime}}\right]$ having a degree 3 in $B P^{\prime}$ is saturated.

1.1.2 If such a matching is found, consider the decomposition $B P_{1, k}^{\prime}$ and $B P_{2, k}^{\prime}$ of $B P^{\prime}$ induced by $E_{k}^{\prime} \cup M_{k}$ and $E^{\prime} \backslash\left(E_{j}^{\prime} \cup M_{k}\right)$ respectively;

1.1.3 Color $B P_{1, k}^{\prime}$ with one color $M_{1}^{k}$;

1.1.4 Color greedily $B P_{2, k}^{\prime}$ with two colors $M_{2}^{k}$ and $M_{3}^{k}$;

1.1.5 Define $\mathcal{S}_{2}^{k}=\left(M_{1}^{k}, M_{2}^{k}, M_{3}^{k}\right)$ the edge coloring of $B P^{\prime}$;

2 Output $\left\{\mathcal{S}_{2}^{k}: k=1, \ldots, m^{\prime}\right\}$

Lemma 4.3 If $\mathcal{S}=\left(M_{1}, M_{2}, M_{3}\right)$ is an edge coloring of $B P^{\prime}$, then we have $\operatorname{val}\left(\mathcal{S}_{2}^{i_{2}-1}\right) \leqslant w\left(M_{1}\right)+$ $2 w\left(M_{2}\right)$.

SOL3

1 For $k=m^{\prime}$ downto 1 do

1.1 Determine if there is a matching $M_{k}$ in $B P^{\prime}\left[\overline{E_{k}^{\prime}}\right]$ such that each node of degree 3 in $B P^{\prime}$ is saturated.

1.2 If such a matching is found, consider the decomposition $B P_{1, k}^{\prime}$ and $B P_{2, k}^{\prime}$ of $B P^{\prime}$ induced by $M_{k}$ and $E^{\prime} \backslash M_{k}$ respectively;

1.3 Color $B P_{1, k}^{\prime}$ with one color $M_{3}^{k}$;

1.4 Color greedily $B P_{2, k}^{\prime}$ with two colors $M_{1}^{k}$ and $M_{2}^{k}$;

1.5 Define $\mathcal{S}_{3}^{k}=\left(M_{1}^{k}, M_{2}^{k}, M_{3}^{k}\right)$ the edge coloring of $B P^{\prime}$; 
2 Output $\left\{\mathcal{S}_{3}^{k}: k=1, \ldots, m^{\prime}-1\right\}$

Lemma 4.4 If $\mathcal{S}=\left(M_{1}, M_{2}, M_{3}\right)$ is an edge coloring of $B P^{\prime}$, then we have $\operatorname{val}\left(\mathcal{S}_{3}^{i_{3}-1}\right) \leqslant 2 w\left(M_{1}\right)+$ $w\left(M_{3}\right)$.

Theorem 4.5 BIPARTITEEDGECOLOR is a $\frac{7}{6}$ approximation for MIN WEIGHTED EDGE COLORING in bipartite graphs with maximum degree 3.

Proof : Let $\mathcal{S}^{*}=\left(M_{1}^{*}, \ldots, M_{5}^{*}\right)$ with $w\left(M_{1}^{*}\right) \geqslant \ldots \geqslant w\left(M_{5}^{*}\right)$ be an optimal weighted edge coloring of BP. Denote by $i_{k}^{*}$ the smallest index of an edge in $M_{k}^{*}\left(i_{k}^{*}=m+1\right.$ if the color is empty). Consider the iteration of BIPARTITEEDGECOLOR corresponding to the cases $i=i_{5}^{*}-1$ and $j=i_{4}^{*}-1$. Then, applying Lemma 4.2 , we produce on $B P^{\prime}=B P\left[E_{i}\right]$ an edge coloring of weight at most $w\left(M_{1}^{*}\right)+w\left(M_{2}^{*}\right)+2 w\left(M_{3}^{*}\right)$. Then the greedy coloring of the edges of $\overline{E_{i}}$ produces a coloring $\mathcal{S}_{1}^{\prime}$ of weight $\operatorname{val}\left(\mathcal{S}_{1}^{\prime}\right) \leqslant w\left(M_{1}^{*}\right)+w\left(M_{2}^{*}\right)+2 w\left(M_{3}^{*}\right)+w\left(M_{5}^{*}\right)$. Applying the same arguments on Lemma 4.3 and Lemma 4.4 , we produce two solutions $\mathcal{S}_{2}^{\prime}$ and $\mathcal{S}_{3}^{\prime}$ respectively satisfying $\operatorname{val}\left(\mathcal{S}_{2}^{\prime}\right) \leqslant$ $w\left(M_{1}^{*}\right)+2 w\left(M_{2}^{*}\right)+2 w\left(M_{4}^{*}\right)$ and $\operatorname{val}\left(\mathcal{S}_{3}^{\prime}\right) \leqslant 2 w\left(M_{1}^{*}\right)+w\left(M_{3}^{*}\right)+2 w\left(M_{4}^{*}\right)$.

Notice that if there is an empty color produced by one of the algorithms SOL $i$, then the bounds are still valid. The convex combination of these 3 values with coefficients $\frac{3}{6} \times \operatorname{val}\left(\mathcal{S}_{1}^{\prime}\right), \frac{2}{6} \times \operatorname{val}\left(\mathcal{S}_{2}^{\prime}\right)$ and $\frac{1}{6} \times \operatorname{val}\left(\mathcal{S}_{3}^{\prime}\right)$ gives the expected result.

\section{$5 \quad$ Weighted node coloring in split graphs}

The split graphs are a class of graphs related to bipartite graphs. Formally, $G=\left(K_{1}, V_{2} ; E\right)$ is a split graph if $K_{1}$ is a clique of $G$ with size $\left|K_{1}\right|=n_{1}$ and $V_{2}$ is an independent set with size $\left|V_{2}\right|=n_{2}$. So, a split graph can be viewed as a bipartite graph where the left set is a clique. Since split graphs forms a subclass of perfect graphs, the node coloring problem on split graphs is polynomial. On the other hand, in [4], it is proved that the weighted node coloring problem is strongly NP-complete in split graphs, even if the weights take only two values. Thus, we deduce that there is no fully polynomial time approximation scheme in such a class of graphs. Here, we propose a polynomial time approximation scheme using structural properties of optimal solutions. An immediate observation of split graphs is that any optimal node coloring $\mathcal{S}^{*}=\left(S_{1}^{*}, \ldots, S_{\ell}^{*}\right)$ satisfies $\left|K_{1}\right| \leqslant \ell \leqslant\left|K_{1}\right|+1$ and any color $S_{i}^{*}$ is a subset of $V_{2}$ with possibly one node of $K_{1}$. In particular, for any optimal node coloring $\mathcal{S}^{*}=\left(S_{1}^{*}, \ldots, S_{\ell}^{*}\right)$, there exists at most one index $i\left(\mathcal{S}^{*}\right)$ such that $S_{i\left(\mathcal{S}^{*}\right)}^{*} \cap K_{1}=\emptyset$.

Lemma 5.1 There is an optimal weighted node coloring $\mathcal{S}^{*}=\left(S_{1}^{*}, \ldots, S_{\ell}^{*}\right)$ with $w\left(S_{1}^{*}\right) \geqslant \ldots \geqslant$ $w\left(S_{\ell}^{*}\right)$ and an index $i_{0} \leqslant \ell+1$ such that:

- $\forall j<i_{0} S_{j}^{*}=\left\{v_{j}\right\} \cup\left\{v \in V_{2}: v \notin \cup_{k=1}^{j-1} S_{k}^{*}\right.$ and $\left.\left[v, v_{j}\right] \notin E\right\}$ for some $v_{j} \in K_{1}$.

- $S_{i_{0}}^{*}=V_{2} \backslash\left(S_{1}^{*} \cup \ldots \cup S_{i_{0}-1}^{*}\right)$ and $\forall j>i_{0} S_{j}^{*}=\left\{v_{j}\right\}$ for some $v_{j} \in K_{1}$. 
Thus, applying an exhaustive search on all sets $K_{1}^{\prime} \subseteq K_{1}$ with $k=\left|K_{1}^{\prime}\right| \leqslant\left\lceil\frac{1}{\varepsilon}\right\rceil$ and on all bijections from $\{1, \ldots, k\}$ to $K_{1}^{\prime}$, one can find the $k$ heaviest colors of an optimal weighted node coloring and thus, we deduce:

Theorem 5.2 MIN WEIGHTED NODE COLORING admits a polynomial time approximation scheme in split graphs.

\section{References}

[1] C. Berge[1973]. Graphs and hypergraphs. North Holland, Amsterdam.

[2] H. L. Bodlaender, K. Jansen, and G. J. Woeginger[1990]. Scheduling with incompatible jobs. Discrete Appl. Math., 55:219-232.

[3] F. R. K. Chung, A. Gyárfás, Zs. Tuza and W. T. Trotter [1990]. The maximum number of edges in 2K2-free graphs of bounded degree. Discrete Mathematics, 81:129-135.

[4] M. Demange, D. De Werra, J. Monnot and V.Th. Paschos [2002]. Weighted node coloring: when stable sets are expensive. Proc. WG'02 LNCS 2573:114-125.

[5] M. R. Garey and D. S. Johnson [1979]. Computers and intractability. a guide to the theory of NP-completeness. CA, Freeman.

[6] H. Grotzsch [1959]. Ein dreifarbensatz fur dreikreisfreie netze auf der Kugel. Wiss. Z. Martin Luther Univ. Halle-Wittenberg, Math. Naturwiss Reihe, 8:109-120.

[7] D. J. Guan And X. Zhu [1997]. A Coloring Problem for Weighted Graphs. Inf. Process. Lett., $61(2): 77-81$.

[8] R. Hassin and J. Monnot [2004]. The maximum saving partition problem. Op. Res. Lett., to appear.

[9] P. L. Hammer, U. N. Peled and X. Sun [1990]. Difference graphs. Discrete Applied Mathematics, 28:35-44.

[10] M. Hujter And Zs. Tuza [1993]. Precoloring extension. II. Graphs classes related to bipartite graphs. Acta Math. Univ. Comeniane, LXII:1-11.

[11] M. Hujter and Zs. Tuza [1996]. Precoloring extension. III. Classes of perfect graphs. Combin. Probab. Comput., 5:35-56.

[12] D. KÖNIG [1916]. Über graphen und iher anwendung auf determinantentheorie und mengenlehre. Math. Ann., 77:453-465.

[13] J. Kratochvil [1993]. Precoloring extension with fixed color bound. Acta Math. Univ. Comen., 62:139-153. 
[14] D. Lichtenstein [1982]. Planar formulae and their uses. SIAM J. Comput., 11(2):329-343.

[15] D. Marx [2004]. NP-completeness of list coloring and precoloring extension on the edges of planar graphs. Technical report available to http://www.cs.bme.hu/ dmarx/publications.html.

[16] C. H. Papadimitriou[1994]. Computational Complexity. Addison Wesley. 